\title{
Engineering human intestinal organoids with a functional ENS
}

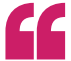

...an obvious

is to model

disease... use of HIOs

Stem-cell-derived human intestinal tissue can be engineered with a functional enteric nervous system (ENS), according to a new study published in Nature Medicine. These ENS-containing human intestinal organoids (HIOs) were then used to model and understand aspects of the biology of the motility disorder Hirschsprung disease.

This new research paper was a follow-up of previous studies that demonstrated generation of HIOs in vitro and maturation after engraftment in vivo, except this time the key focus was on development of a functional ENS in the HIOs. "The tissues [from previous work] had epithelial and smooth muscle, but no enteric nerves, and thus did not exhibit any motility or contractility," explains author James Wells. "This aspect was a major deficiency, given that one of our long-term goals is to engineer fully functional intestinal tissue," he adds, noting that an obvious

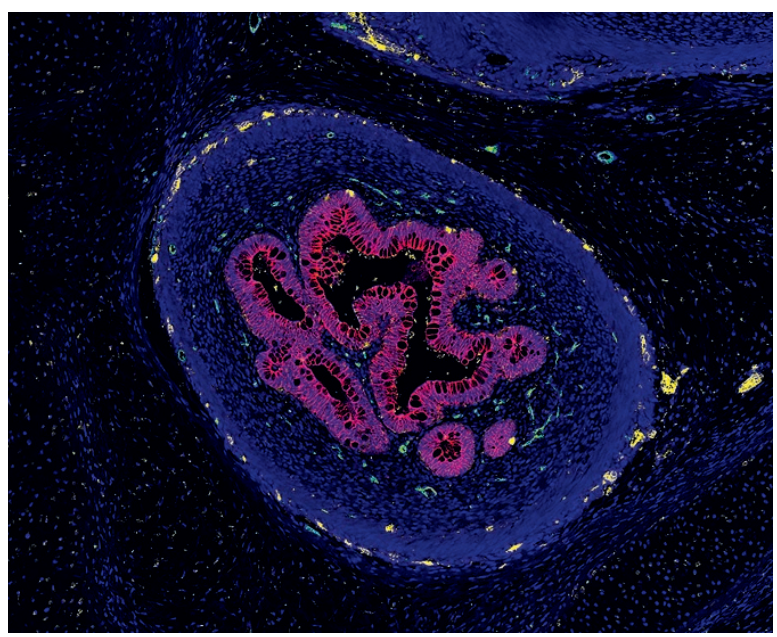

Human intestinal organoid with enteric nervous system, Image courtesty of M. M. Mahe, M. A. Helmrath and J. M. Wells. use of HIOs is to model disease, but without having an ENS component these organoids would not be useful in the context of motility disorders such as Hirschsprung disease.

Wells and colleagues used a tissue engineering approach with embryonic and induced pluripotent stem cells (PSCs) to generate intestinal tissue with enteric nerves. First, they generated human vagal neural crest cells (NCCs, the precursors of the ENS) derived from PSCs, which were then incorporated into HIOs by mechanical aggregation of midgut-hindgut spheroids. These NCCs recombined with HIOs in vitro, then migrated into the mesenchyme where they differentiated into neurons and glial cells. Crucially, neuronal activity was recorded in vitro, as evidenced by oscillations in calcium efflux.

The investigators then engrafted the ENS-containing HIOs into mice. After 6-10 weeks of growth in vivo, the HIOs became vascularized, growing to $\sim 1-3 \mathrm{~cm}$ in diameter and forming mature intestinal tissue (with villi, intestinal crypts and functional intestinal stem cells). Moreover, these engrafted ENS-containing HIOs matured into structures similar to the myenteric and submucosal plexus, alongside the generation of neurons and glial cells that were organized into ganglionated structures in close proximity to smooth muscle fibres. Importantly, the researchers demonstrated ENS-mediated contractile activity in the engrafted HIOs plus ENS, with functional interstitial cells of Cajal (considered the pacemaker cells of the gut) and sustained waves of contraction recorded after electrical field stimulation.
Lastly, Wells and co-workers used their engineered innervated intestinal tissue to investigate the cellular and molecular basis of Hirschsprung disease. In particular, they examined the effects of $P H O X 2 B$ mutations, which result in complete aganglionosis of the bowel. Induced PSCs containing various $P H O X 2 B$ alleles and mutations $-\mathrm{PHOX} 2 \mathrm{~B}^{+/+}, \mathrm{PHOX} 2 \mathrm{~B}^{+/ Y 14 X}$ and $P H O X 2 B^{Y 14 X / Y 14 X}$ - were generated and then differentiated into vagal NCCs to be recombined with HIOs. Transcriptional analysis of organoids demonstrated distinct clustering of transcripts according to genotype. Mutant $\mathrm{PHOX} 2 \mathrm{~B}$ organoids had altered gene expression, such as reduced expression of NCCrelated genes, reduced muscle cell development and increased central nervous system markers. Furthermore, engrafted HIOs plus $P H O X 2 B$ mutant ENS grew poorly, had few neurons and had poorly developed smooth muscle.

The researchers will continue using these ENS-containing HIOs in models of health and disease to study organ development, enteric pathogens and mechanisms of paediatric diseases such as Hirschsprung disease. "My collaborator, and cocorresponding author Dr Helmrath, a paediatric surgeon, is developing transplant models using these more functional human intestinal tissues to study how they function when transplanted into the luminal stream of animals," says Wells.

Katrina Ray

ORIGINAL ARTICLE Workman, M. J. et al. Engineered human pluripotent-stem-cell-derived intestinal tissues with a functional enteric nervous system. Nat. Med. http://dx.doi.org/10.1038/ nm.4233 (2016) 